\title{
Overview of Structural Reliability Analysis Methods - Part II: Sampling Methods
}

\author{
ChangWu HUANG ${ }^{1,{ }^{*}}$, Abdelkhalak El Hami ${ }^{1}$, Bouchaïb Radi ${ }^{2}$ \\ ${ }^{1}$ Normandie Université, INSA Rouen, LOFIMS, 76000 Rouen, France. \\ "Corresponding author: ChangWu.Huang.PRC@gmail.com \\ ${ }^{2}$ LIMII, FST Settat, BP: 577, Route de Casa, Settat, Morocco.
}

ABSTRACT. In Part II of the overview of structural reliability analysis methods, the category of sampling methods is reviewed. The basic Monte Carlo simulation is the foundation for sampling methods of reliability analysis. Sampling methods can evaluate the failure probability defined by both explicit and implicit performance function. With sufficient number of samples, simulation methods can give accurate results. However, for complex problem the computational cost is expensive. Thus, based on variance reduction techniques, some variants of basic Monte Carlo simulation method are proposed to reduce the computational cost. Monte Carlo simulation and its variants, including importance sampling, adaptive sampling, Latin hypercube sampling, directional simulation, and subset simulation, are presented and summarized in this paper.

KEYWORDS. Reliability Analysis, Sampling Methods, Monte Carlo simulation, Variance Reduction.

\section{Introduction}

Reliability analysis, which offers the theoretical framework for considering uncertainties in engineering decision scheme [1], plays important roles in modern design. Our work devotes to provide a comprehensive overview and some valuable remarks on reliability analysis methods for researchers and engineers. In Part I of the overview, the local reliability methods are reviewed completely.

In this paper, the Part II of the overview, we focus on sampling methods for calculating the probability of failure in reliability problems. In Section 2, the basic Monte Carlo simulation (MCS) methods and several popular invariants of MCS are detailed. Subsequently, a typical example is presented in Section 3 to illustrate some sampling methods and local reliability methods. In the end, a brief summary is given in Section 4.

\section{Sampling Methods}

Sampling methods (or simulation method) have a long history in probabilistic analysis. The primary goal of reliability analysis is to calculate the failure probability defined by

$$
\begin{aligned}
p_{\mathrm{f}} & =\mathrm{P}(G(\mathbf{X}) \leq 0)=\int_{G(\mathbf{X}) \leq 0} f_{X}(\mathbf{x}) \mathrm{d} \mathbf{x} \\
& =\mathrm{P}(H(\mathbf{U}) \leq 0)=\int_{H(\mathbf{U}) \leq 0} \phi_{U}(\mathbf{u}) \mathrm{d} \mathbf{u}
\end{aligned}
$$

where $\mathrm{P}(\cdot)$ is the probability operator, $\mathbf{x}$ is a random vector that represents the design variables, $G(\mathbf{x})$ is the performance function defined in $\mathbf{X}$-space, $f_{X}(\mathbf{x})$ is the joint probability density function (PDF) of random vector $\mathbf{x}, H(\mathbf{U})$ is the performance function in $\mathbf{U}$-space, and $\phi_{U}(\mathbf{u})$ is the standard multidimensional Gaussian density function. More details about this can be found in Part I of our work.

By using sampling methods, the failure probability defined by Equation (1) in for both explicit and implicit performance function can be evaluated. The crude (or basic) Monte Carlo simulation (MCS) is the foundation for this kind of reliability methods. Other variants, which introduced variance reduction techniques, are proposed in order to reduce the computational cost of crude MCS. 


\subsection{Monte Carlo Simulation (MCS) Method}

Monte Carlo Simulation (MCS) is a widely used method for numerically computing integrals and expected values. It is therefore adopted in reliability problems for estimating the failure probability. The basis for simulation techniques is well illustrated by rewriting the failure probability integral in Equation (1) by changing the domain of integration to the entire space using an indicator function. The rewritten integral equation is

$$
p_{\mathrm{f}}=\int_{G(X) \leq 0} f_{X}(\mathbf{x}) \mathrm{d} \mathbf{x}=\int_{-\infty}^{\infty} \mathbb{I}[G(\mathbf{x})] f_{X}(\mathbf{x}) \mathrm{d} \mathbf{x}
$$

where $\mathbb{I}[G(\mathbf{x})]$ is an indicator function of the performance function, which is defined as

$$
\mathbb{I}[G(\mathbf{x})]= \begin{cases}1, & \text { if } G(\mathbf{x}) \leq 0 \\ 0, & \text { if } G(\mathbf{x})>0\end{cases}
$$

By using the above defined indicator function, the integration domain is changed from the failure domain $G(\mathbf{x}) \leq 0$ to the entire sample space. From Equation (2), we can see that the failure probability is the expected value of $\mathbb{I}[G(\mathbf{x})]$, i.e., we can have

$$
p_{\mathrm{f}}=\int_{-\infty}^{\infty} \mathbb{I}[G(\mathbf{x})] f_{X}(\mathbf{x}) \mathrm{d} \mathbf{x}=\mathbb{E}(\mathbb{I}[G(\mathbf{x})])
$$

where $\mathbb{E}(\cdot)$ denotes the expected value. Therefore, we can estimate the failure probability by the following procedure. First, $N$ realizations of the random variables $\mathbf{x}^{(i)}, i=1, \cdots, N$ are sampled according to the probability distribution of $\mathbf{X}$. Then the performance functions are evaluated at these input values. Finally, the number of the points in failure domain among these $N$ samples are countered in $N_{\mathrm{f}}$. Consequently, we have

$$
\hat{p}_{\mathrm{f}}=\frac{1}{N} \sum_{i=1}^{N} \mathbb{I}\left[G\left(\mathbf{x}^{(i)}\right)\right]=\frac{N_{\mathrm{f}}}{N}
$$

is an unbiased estimator of the failure probability $p_{\mathrm{f}}$, i.e., $\mathbb{E}\left[\hat{p}_{\mathrm{f}}\right]=p_{\mathrm{f}}$. The coefficient of variation of the estimator $\hat{p}_{\mathrm{f}}$ is

$$
\operatorname{Cov}\left[\hat{p}_{\mathrm{f}}\right]=\frac{\sigma_{\hat{p}_{\mathrm{f}}}}{\mathbb{E}\left[\hat{p}_{\mathrm{f}}\right]}=\frac{\sqrt{\operatorname{Var}\left[\hat{p}_{\mathrm{f}}\right]}}{\mathbb{E}\left[\hat{p}_{\mathrm{f}}\right]}=\sqrt{\frac{1-p_{\mathrm{f}}}{N p_{\mathrm{f}}}}
$$

where $\operatorname{Var}\left[\hat{p}_{\mathrm{f}}\right]=p_{\mathrm{f}}\left(1-p_{\mathrm{f}}\right) / N$ is the variance of $\hat{p}_{\mathrm{f}}$. The coefficient of variation $\operatorname{Cov}\left[\hat{p}_{\mathrm{f}}\right]$ measures the convergence rate of the Monte Carlo simulation results. According to Equation (6), for an objective $\operatorname{Cov}\left[\hat{p}_{\mathrm{f}}\right]=0.1$ and a probability $p_{\mathrm{f}}=10^{-m}$, approximately $N=10^{m+2}$ samples are needed.

The MCS is a simple sampling (simulation) method is simple to implement and can be applied to almost all reliability problems, at any desired accuracy [2]. However, the performance function needs to be evaluated a large number of times with randomly sampled input values of the basic variables, which can be time consuming and expensive for problems with implicit performance functions or (and) high reliability (i.e. low failure probability).

In order to enhance the efficiency of MCS, several more-efficient sampling methods, such as Importance Sampling, Directional Simulation, and Subset Sampling, have been developed [3]. They form the kernel of advanced sampling methods for structural reliability.

\subsection{Importance Sampling}

The basic idea of importance sampling (IS) is to focus on the region(s) of "importance" so as to save computational resources [4]. The importance region(s) in reliability problem can be seen as the failure 
region. The concept underlying the importance sampling (IS) method is to replace the original PDF $f_{X}(\mathbf{x})$ with an IS distribution $h_{V}(\mathbf{x})$ such that a large number of samples lies in the "important region" of the sample space, i.e., the failure region in reliability problem [5]0. Accordingly, the failure probability of Equation (2) can be rewritten as

$$
p_{\mathrm{f}}=\int_{-\infty}^{\infty} \mathbb{I}[G(\mathbf{v})] \frac{f_{X}(\mathbf{v})}{h_{V}(\mathbf{v})} h_{V}(\mathbf{v}) \mathrm{d} \mathbf{v}
$$

where $h_{V}(\mathbf{v})$ is called the importance sampling probability density function (PDF) or instrumental probability density function and $\int_{-\infty}^{\infty} h_{v}(\mathbf{v}) \mathrm{d} v=1$. This transformation implies that the estimator of the failure probability in Equation (5) becomes

$$
\hat{p}_{\mathrm{f}}=\frac{1}{N} \sum_{i=1}^{N} \mathbb{I}\left[G\left(\mathbf{v}^{(i)}\right)\right] \frac{f_{X}\left(\mathbf{v}^{(i)}\right)}{h_{V}\left(\mathbf{v}^{(i)}\right)}
$$

where $\mathbf{v}^{(i)}, i=1,2, \cdots, N$ are the samples generated from $h_{V}(\cdot)$ instead of $f_{X}(\cdot)$ as before. The variance of the estimate of importance sampling $\hat{p}_{\mathrm{f}}$ is given by:

$$
\begin{aligned}
\operatorname{Var}\left[\hat{p}_{\mathrm{f}}\right] & =\frac{1}{N} \operatorname{Var}\left[\mathbb{I}[G(\mathbf{v})] \frac{f_{X}(\mathbf{v})}{h_{V}(\mathbf{v})}\right] \\
& =\frac{1}{N}\left(\int \frac{\mathbb{I}[G(\mathbf{v})] f_{X}^{2}(\mathbf{v})}{h_{V}^{2}(\mathbf{v})} f_{X}(\mathbf{v}) \mathrm{d} \mathbf{v}-p_{\mathrm{f}}^{2}\right)
\end{aligned}
$$

It can be seen that a good choice for $h_{V}(\mathbf{v})$ can produce smaller variance of $\hat{p}_{\mathrm{f}}$ than that from crude Monte Carlo simulation method. Conversely, the variance can actually be increased when a very poor choice of $h_{v}(\mathbf{v})$ is used. By minimizing the above variance, the optimal importance sampling PDF can be given as [6]:

$$
h_{V}^{*}(\mathbf{x})=\frac{\mathbb{I}[G(\mathbf{x})] f_{X}(\mathbf{x})}{p_{\mathrm{f}}}= \begin{cases}\frac{1}{p_{\mathrm{f}}} f_{X}(\mathbf{x}) & \text { for } G(\mathbf{x}) \leq 0 \\ 0 & \text { for } G(\mathbf{x})>0\end{cases}
$$

However, this optimal importance sampling PDF is ineffective in practice, because it requires knowledge of $p_{\mathrm{f}}$ priori. Therefore, in practical applications of IS, the optimal importance sampling PDF is approximately obtained and used. In this point, the importance sampling methods in reliability analysis that have been proposed and widely used in the literatures can be grouped into: importance sampling at the design point and adaptive importance sampling.

\subsubsection{Importance Sampling (IS) at the Design Point}

For reliability problems, the region(s) of "importance" is the failure domain, more particularly, the region of greatest probability density within the failure domain. The design point $\mathbf{u}^{*}$, which locates on the limit state and has the minimal distance from the origin in the standard normal space, is considered to be the point with the highest probability density among all realizations in the failure domain $H(\mathbf{u}) \leq 0$. Accordingly, in early applications of the importance sampling concept in structural reliability, the density $h_{V}(\mathbf{v})$ was posed over the design point and $h_{V}(\mathbf{v})$ was simply taken as a multi-dimensional Gaussian distribution. This approach is known as the importance sampling at the design point, which is illustrated in Figure 1.

The search of the design point, apparently, is the basis for this approach. The algorithm of finding the design point $\mathbf{u}^{*}$ is detailed in design point-based methods in Section 3.2 in Part I of this overview. Once the design point $\mathbf{u}^{*}$, whose corresponding point in $\mathbf{X}$-space is denoted by $\mathbf{x}^{*}$, is identified, the 
importance sampling density function $h_{V}(\mathbf{v})$ is chosen as a multivariate Gaussian distribution with the statistical moments $\mathbb{E}(\mathbf{V})=\mathbf{x}^{*}$ and $\boldsymbol{\Sigma}_{V V}=\boldsymbol{\Sigma}_{X X}\left(\boldsymbol{\Sigma}_{V V}\right.$ is the covariance matrix for importance sampling density and $\Sigma_{X X}$ is the covariance matrix of basic random vector $\left.\mathbf{x}\right)$ :

$$
h_{V}(\mathbf{v})=\frac{1}{\sqrt{(2 \pi)^{k}\left|\boldsymbol{\Sigma}_{V V}\right|}} \exp \left[-\frac{1}{2}\left(\mathbf{v}-\mathbf{x}^{*}\right)^{\mathrm{T}} \boldsymbol{\Sigma}_{V V}^{-1}\left(\mathbf{v}-\mathbf{x}^{*}\right)\right]
$$

Then samples generated according to this density function $h_{V}(\mathbf{v})$ are used to estimate the probability of failure.

The importance sampling at the design point is simple to implement and usually works efficiently. However, since this approach depends on the design point, it may fail when the limit state function is highly nonlinear or give inaccurate results. Moreover, if there are multiple local minima in the search of the design point, this approach may not work well.

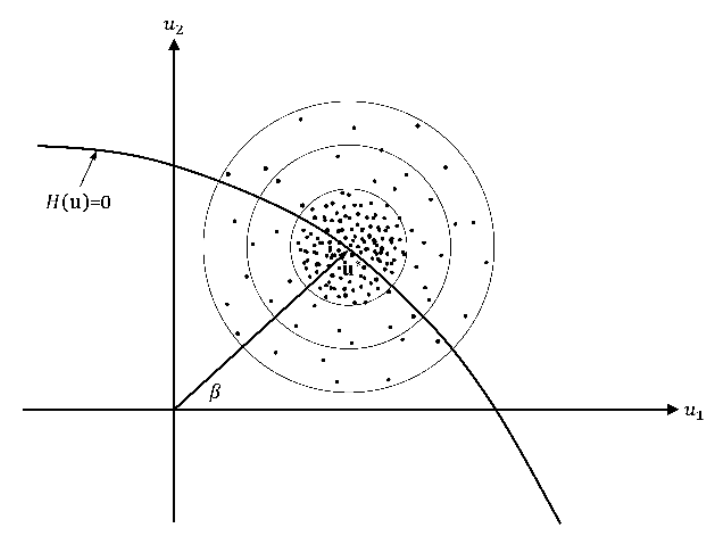

Figure 1. Importance Sampling at the Design Point

\subsubsection{Adaptive Sampling (AS)}

Based on the idea that the efficiency of simulation methods can be increased by maximizing the number of the samples in failure domain, it would be efficient to choose the importance sampling density as the origin density conditional on the failure domain [7], i.e.,

$$
h_{V}(\mathbf{v})=f_{X}(\mathbf{v} \mid \mathbf{v} \in \mathcal{F})
$$

However, this function is not known in advance. In adaptive sampling method, the desired conditional distribution is estimated by statistics of the points in the failure domain from previous simulations [8]. The new distribution is then used to carry out the subsequent sampling. The ideal conditional density cannot be met strictly. It is possible that Equation (12) can be satisfied at least in terms of the first and second moments. In other words, the mean and standard deviation (or covariance) of the conditional density function are calculated from the sampling points in the failure domain, i.e.,

$$
\begin{aligned}
& \mathbb{E}_{h}[\mathbf{V}]=\mathbb{E}_{f}[\mathbf{V} \mid \mathbf{v} \in \mathcal{F}] \\
& \mathbb{E}_{h}\left[\mathbf{V} \mathbf{V}^{\mathrm{T}}\right]=\mathbb{E}_{f}\left[\mathbf{V} \mathbf{V}^{\mathrm{T}} \mid \mathbf{v} \in \mathcal{F}\right]
\end{aligned}
$$

The indices $h$ and $f$ refer to the respective joint densities $h_{V}(\mathbf{v})$ and $f_{X}(\mathbf{v})$. The first set of samples is performed using the importance sampling at the design point. Then the right-hand sides of the Equation (13) can be estimated. These two values are used to adapt $h_{V}(\mathbf{v})$ for the next run. In terms of these two statistical moments (mean and covariance), a joint Gaussian distribution is uniquely determined and this distribution is chosen for $h_{V}(\mathbf{v})$ in subsequent sampling. Through the iterative adaptive algorithm, Equation (12) can be satisfied in terms of first and second moments. 


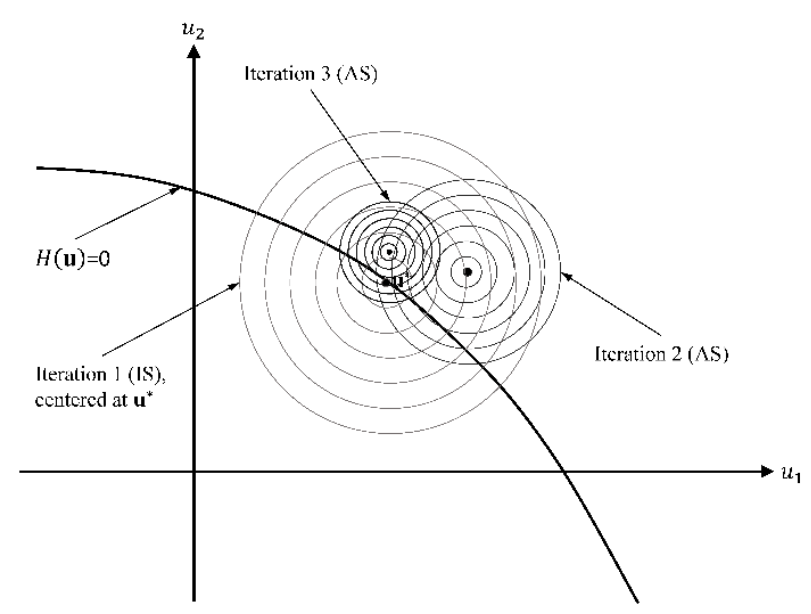

Figure 2. Iterations of Adaptive Sampling

\subsection{Latin Hypercube Sampling (LHS)}

Generally, in simulation methods, the random sampling method is adopted to generate samples. In order to improve the efficiency of basic MCS, another sampling method called Latin Hypercube Sampling (LHS) was developed [9]. Latin hypercube sampling is a widely-used method to generate controlled random samples and its basic idea is to make sampling point distribution close to the probability density function (PDF).

LHS uses a stratified sampling scheme to improve the coverage of the input space. The stratification is implemented by dividing the vertical axis on the graph of the cumulative distribution function (CDF) $F_{X_{j}}(\cdot)$ of a random variable $X_{j}$ into $N$ non-overlapping intervals of equal length, where $N$ is the number of samples. Through the inverse of $\mathrm{CDF}_{X_{j}}^{-1}(\cdot)$, these $N$ intervals divide the horizontal axis into $N$ equiprobable, but not necessarily equal-length, intervals. Thus, the axis of $X_{j}$ (horizontal axis) has been stratified into $N$ equiprobable and non-overlapping intervals. That is to say the range of random variable $X_{j}$ is divided into $N$ equally probable intervals.

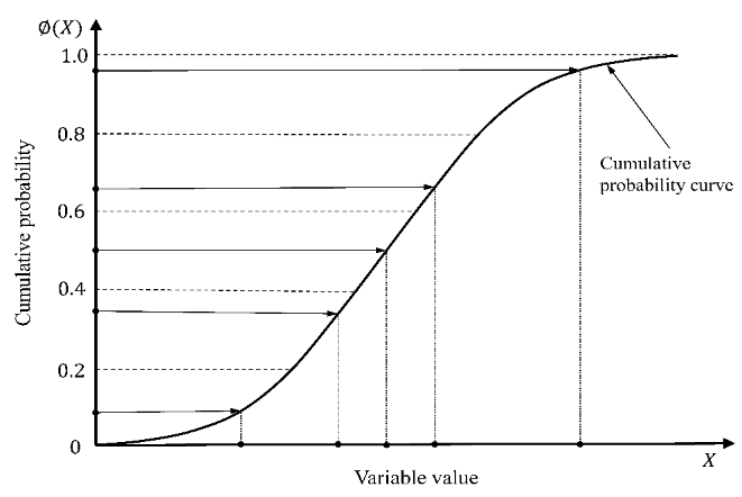

Figure 3. Evenly partition CDF and Equiprobable Intervals of Random Variable

Then, randomly pick up one value within each of the $N$ intervals on the vertical axis defined before. With $F_{X_{j}}^{-1}(\cdot)$, these selected values are mapped on horizontal axis such that exactly one value in each equally probable intervals of $X_{j}$. In this way, $N$ samples of one-dimensional random variable $X_{j}$ are generated by LHS (seen in Figure 3). In order to generate $N$ samples of a $k$-dimensional random vector $\mathbf{X}=\left[X_{1}, \cdots, X_{k}\right]^{\mathrm{T}}, N$ samples of each component of $\mathbf{x}$ is firstly generated by LHS as previously described, then the $N$ values of $X_{1}$ are paired in a random manner with values of $X_{2}$, sequentially, these pairs are then paired similarly with values of $X_{3}$ and so on, until $N$ values of $k$ components of $\mathbf{x}$ are formed. The $N$ samples of random vector $\mathbf{x}$ form an $k \times N$ matrix whose $j$-th row contains the LHS for the 
component $X_{j}$. A random process is used to ensure the random ordering (pairing) of the values within each row of this matrix. This mixing process serves to emulate the paring of observations in a simple Monte Carlo process.

LHS ensures that the entire range of each input variable is completely covered. It has been shown that LHS is more efficient than simple random sampling in a large range of conditions [10]. LHS has been widely used in design of experiments and sampling methods for reliability problems owing to its efficiency.

\subsection{Directional Simulation}

Directional Simulation (DS) reduces the dimension of probability integral by identifying a set of directions for integration and estimating the probability as a weight average of the directional integrals [11]. It is based on the concept of conditional probability and it also exploits the symmetry of the standard normal space $\mathbf{U}[12]$. The key idea of directional simulation is firstly seeking a set of directions in $\mathbf{U}$-space and then perform the reliability analysis as a sequence of one-dimensional integrations in each direction.

The $n$-dimensional normal vector $\mathbf{U}$ can be expressed as $\mathbf{U}=R \mathbf{A}, R \geq 0$, where $\mathbf{A}$ is a random unit vector distributed on the $n$-dimensional unit sphere $\Omega^{n}$, and $R^{2}(R=\|\mathbf{U}\|)$ is a chi-square $\left(\chi^{2}\right)$ distributed random variable with $n$ degrees of freedom and independent of $\mathbf{A}$. The failure probability $p_{\mathrm{f}}$ can be expressed as

$$
p_{\mathrm{f}}=\int_{a \in \Omega^{\prime \prime}} P[H(R \mathbf{A}) \leq 0 \mid \mathbf{A}=\mathbf{a}] f_{A}(\mathbf{a}) \mathrm{d} \mathbf{a}
$$

where $f_{\mathbf{A}}(\mathbf{a})$ is the uniform probability density function of $\mathbf{A}$ on the unit sphere $\Omega^{n}$.

Practically, a sequence of $N$ random direction vectors $\mathbf{a}^{(i)}=\mathbf{u}^{(i)} /\left\|\mathbf{u}^{(i)}\right\|, i=1, \cdots, N$, is generated fist, then $r_{i}$ which are the solution of $G\left(r \mathbf{a}^{(i)}\right)=0$ are found iteratively, the failure probability is estimated as

$$
\hat{p}_{\mathrm{f}}=\frac{1}{N} \sum_{i=1}^{N} P\left[H\left(r \mathbf{a}^{(i)}\right) \leq 0\right]=\frac{1}{N} \sum_{i=1}^{N}\left[1-\chi_{n}^{2}\left(r_{i}\right)\right]
$$

where $\chi_{n}^{2}[\cdot]$ is the chi-square CDF with $n$ degrees of freedom.

Directional simulation eliminates the limitations in situations of nonlinearity of the limit state function or multiple design points. Furthermore, importance directional simulation [13][14], which uses the importance sampling technique to concentrate the direction vectors in the regions of interest, has also proposed for reliability analysis.

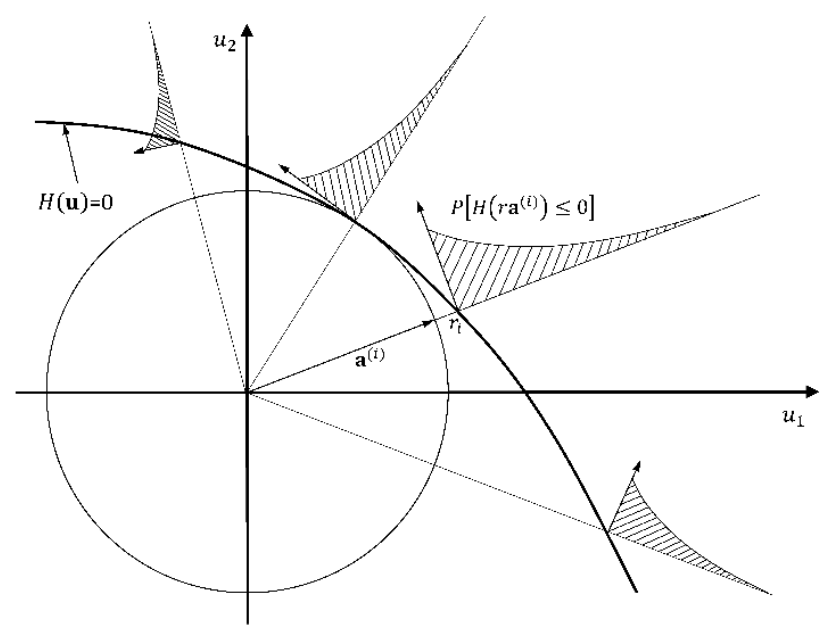

Figure 4. Directional Simulation 


\subsection{Subset Simulation}

Subset Simulation (SS) is a new simulation approach to efficiently deal with small failure probabilities encountered in reliability analysis problems. The underlying idea of subset simulation is to express the (small) failure probability as a product of (larger) probabilities conditional on some intermediate events [15]. This converts the simulation of a rare event into a sequence simulation of more frequent events.

For the target failure event $F$ in reliability problem, let $F_{1} \supset F_{2} \supset \cdots \supset F_{m}=F$ be a decreasing sequence of intermediate events, so that $F_{k}=\bigcap_{i=1}^{k} F_{i}, k=1,2, \cdots, m$. For example, a general failure event $F$ in structural reliability is defined as $F=\{\mathbf{x} \mid g(\mathbf{x}) \leq 0\}$, accordingly, a sequence of intermediate events can simply be defined as $F_{i}=\left\{\mathbf{x} \mid g(\mathbf{x}) \leq C_{i}\right\}$, where $C_{1}>C_{2}>\cdots>C_{m}=0$. By sequentially conditioning on the event $F_{i}$, the target failure probability $p_{\mathrm{f}}$ can be expressed as

$$
p_{\mathrm{f}}=p\left(F_{m}\right)=p\left(F_{1}\right) \prod_{i=1}^{m-1} p\left(F_{i+1} \mid F_{i}\right)
$$

We can see that even if $p_{\mathrm{f}}$ is small, the conditional probabilities $\left\{p\left(F_{i+1} \mid F_{i}\right), i=1,2, \cdots, m-1\right\}$ and $p\left(F_{1}\right)$ in Equation (16) can be made sufficiently large by appropriately choosing $m$ and the intermediate events $\left\{F_{i}, i=1,2, \cdots, m-1\right\}$. This overcomes the difficulty of crude Monte Carlo simulation in small failure probability situations and makes subset simulation an efficient simulation method, especially for condition with small failure probability. For example, considering $p_{\mathrm{f}} \approx 10^{-5}$ and choosing $m=4$ intermediate events such that $p\left(F_{1}\right)$ and $p\left(F_{i+1} \mid F_{i}\right) \approx 0.1, i=1,2,3$, the conditional probabilities can be efficiently evaluated by simulation of the relatively frequent intermediate events, even though the directly simulation of $p_{\mathrm{f}}$ is very computationally expensive [16].

The original idea of subset simulation is to estimate the failure probability $p_{\mathrm{f}}$ by estimating $p\left(F_{1}\right)$ and $p\left(F_{i+1} \mid F_{i}\right), i=1,2, \cdots, m-1$. In simulation, standard MCS can be used to estimate $p\left(F_{1}\right)$, while the conditional probabilities $\left\{p\left(F_{i+1} \mid F_{i}\right), i=1,2, \cdots, m-1\right\}$ are estimated by Markov Chain Monte Carlo (MCMC) simulation method [17].

\section{Illustrative Example}

The example in this subsection is used to illustrate some sampling methods, including MCS, IS and LHS. Additionally, this example is also solved by FORM and SORM. Suppose that the performance function of a structure is defined by

$$
G(\mathbf{X})=X_{2} X_{3} X_{4}-\frac{X_{3}^{2} X_{4}^{2} X_{5}}{X_{6} X_{7}}-X_{1}
$$

where $\mathbf{X}=\left[X_{1}, \cdots, X_{7}\right]^{\mathrm{T}}$ are the basic random variables which are independently normal distributed with mean value vector

$$
\mu_{X}=\left[0.01,0.3,360,2.26 \times 10^{-4}, 0.5,0.12,40\right]^{\mathrm{T}}
$$

and coefficient of variation vector

$$
\operatorname{cov}_{X}=[0.30,0.05,0.10,0.05,0.10,0.05,0.15]^{\mathrm{T}} .
$$

\section{FORM and SORM Solutions}

Firstly, the FORM is performed to solve this problem. The results of FORM are the basis for using SORM and IS based on design point. The reliability index and failure probability of FORM are

$$
\beta=3.4131, \quad p_{\mathrm{f} 1}=\Phi(-\beta)=3.2113 \times 10^{-4}
$$


The design point in $\mathbf{X}$-space is $\mathbf{x}^{*}=[0.0178,0.2875,292.7685,0.0002,0.5019,0.1199,39.6627]^{\mathrm{T}}$

The failure probability calculated by SORM, based on FORM results, is $p_{\mathrm{f} 2}=3.3869 \times 10^{-4}$

\section{Monte Carlo Simulation (MCS)}

In order to illustrate the convergence of Monte Carlo simulation (MCS), different number of samples are used in basic MCS. In order to obtain the coefficient of variation defined in Equation (6), which is usually used to measure the convergence rate of MCS, the exact probability of failure $p_{\mathrm{f}}$ is necessary. Here, the result of a MCS with $10^{8}$ samples is taken as the exact (or true) value of failure probability, that is $p_{\mathrm{f}}=3.387 \times 10^{-4}$. The coefficient of variation $\left(\operatorname{Cov}\left[\hat{p}_{\mathrm{f}}\right]\right)$ computed by Equation (6) and corresponding sample size $\left(N_{\mathrm{MCS}}\right)$ are listed in Table 1.

The MCS solutions $\left(\hat{p}_{\mathrm{f}}\right)$ by the use of different number of samples $\left(N_{\text {MCs }}\right)$ are reported in Table 2 . It is necessary to note that the solution of each certain number of samples is the average probability of 50 independent simulations with the same sampling number. The coefficient of variation $\operatorname{Var}\left[\hat{p}_{\mathrm{f}}\right]$, estimated failure probability $\hat{p}_{\mathrm{f}}$ and the failure probability with \pm 2 standard deviations $\left(\hat{p}_{\mathrm{f}} \pm 2 \sqrt{\operatorname{Var}\left[\hat{p}_{\mathrm{f}}\right]}\right)$ with respect to the number of samples are plotted in Figure 5.

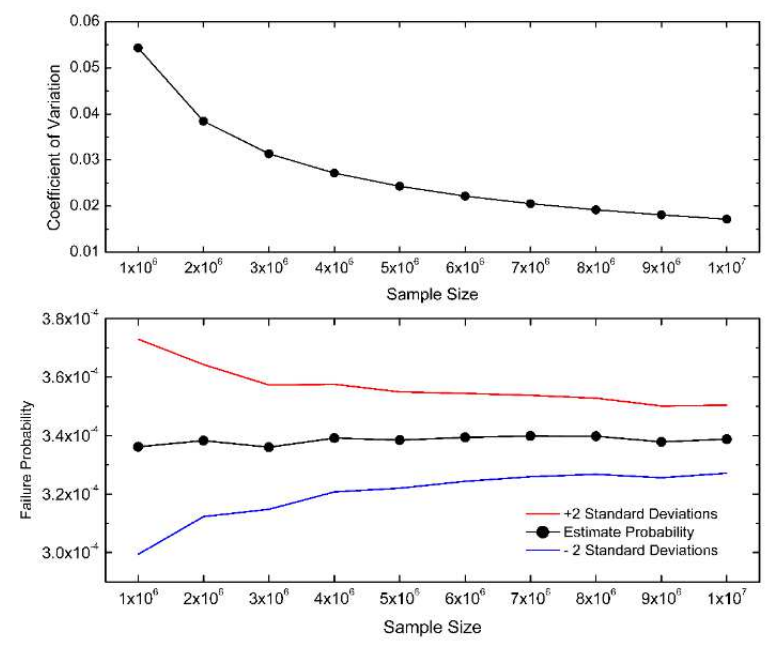

Figure 5. Convergence of Monte Carlo simulations

In Figure 5, it can be seen that the coefficient of variation decreases as the sample size increases. In other words, the solution of MCS converges as the increase of sample size. Therefore, accurate solution can be obtained by using sufficient large sample size. However, the computational cost increase with the augment in sample size. The drawback in MCS can be seen from this.

\section{Latin Hypercube Sampling (LHS)}

Latin Hypercube Sampling (LHS) is also performed to solve this example. The same as MCS, LHS perform with different sample size, from $1 \times 10^{6}$ to $1 \times 10^{7}$, and 50 independent simulations are carried out for each sample size. The average probability from 50 simulations at each sample size are reported in Table 3.

\section{Importance Sampling (IS) at the Design Point}

The importance sampling (IS) using a multivariate Gaussian distribution posed over the design point is applied for this problem. The probability of failure estimated by IS with different sample size are given in Table 4. 
It is obvious that IS can give accurate solution with small sample size than MCS and LHS. The fluctuation of the probability estimated by IS is very small. This is owing to the variance reduction technique in IS. Thus, the use of IS can reduce the computational burden of MCS.

\section{Comparison}

The results from FORM, SORM and above three sampling methods with sample size of $1 \times 10^{7}$ are presented in Table 5.

For this nonlinear problem, the FORM is no capable to give accurate solution due to its first-order approximation of the limit state. While, the SORM improves the FORM and gives sufficient accurate results. Three sampling methods, MCS, LHS and IS, provide accurate estimate of the failure probability. Sampling methods are not restricted by the shape of the limit state and thus can be used for linear or nonlinear problems.

\begin{tabular}{|c|c|c|c|c|c|}
\hline $\boldsymbol{N}_{\text {мсS }}$ & $\mathbf{1 . E}+\mathbf{0 6}$ & $\mathbf{2 . E}+\mathbf{0 6}$ & $\mathbf{3 . E}+\mathbf{0 6}$ & $\mathbf{4 . E}+\mathbf{0 6}$ & $\mathbf{5 . E}+\mathbf{0 6}$ \\
\hline $\operatorname{Cov}\left[\hat{\boldsymbol{p}}_{\mathrm{f}}\right]$ & $5.43 \mathrm{E}-02$ & $3.84 \mathrm{E}-02$ & $3.14 \mathrm{E}-02$ & $2.72 \mathrm{E}-02$ & $2.43 \mathrm{E}-02$ \\
\hline $\boldsymbol{N}_{\text {McS }}$ & $6 . \mathrm{E}+06$ & $7 . \mathrm{E}+06$ & $8 . \mathrm{E}+06$ & $9 . \mathrm{E}+06$ & $1 . \mathrm{E}+07$ \\
\hline $\operatorname{Cov}\left[\hat{\boldsymbol{p}}_{\mathrm{f}}\right]$ & $2.22 \mathrm{E}-02$ & $2.05 \mathrm{E}-02$ & $1.92 \mathrm{E}-02$ & $1.81 \mathrm{E}-02$ & $1.72 \mathrm{E}-02$ \\
\hline
\end{tabular}

Table 1. Coefficient of variance with respect to sample size in MCS

\begin{tabular}{|c|c|c|c|c|c|}
\hline$N_{\mathrm{MCS}}$ & $\mathbf{1 . 0 E}+\mathbf{0 6}$ & $\mathbf{2 . 0 E}+\mathbf{0 6}$ & $\mathbf{3 . 0 E}+\mathbf{0 6}$ & $\mathbf{4 . 0 E}+\mathbf{0 6}$ & $\mathbf{5 . 0 E}+\mathbf{0 6}$ \\
\hline$\hat{p}_{\mathrm{f}}$ & $3.3618 \mathrm{E}-04$ & $3.3828 \mathrm{E}-04$ & $3.3603 \mathrm{E}-04$ & $3.3912 \mathrm{E}-04$ & $3.3846 \mathrm{E}-04$ \\
\hline$\hat{p}_{\mathrm{f}}-2 \sigma_{\hat{p}_{\mathrm{f}}}$ & $2.9938 \mathrm{E}-04$ & $3.1226 \mathrm{E}-04$ & $3.1478 \mathrm{E}-04$ & $3.2072 \mathrm{E}-04$ & $3.2200 \mathrm{E}-04$ \\
\hline$\hat{p}_{\mathrm{f}}+2 \sigma_{\hat{p}_{\mathrm{f}}}$ & $3.7298 \mathrm{E}-04$ & $3.6430 \mathrm{E}-04$ & $3.5728 \mathrm{E}-04$ & $3.5752 \mathrm{E}-04$ & $3.5492 \mathrm{E}-04$ \\
\hline$N_{\mathrm{MCS}}$ & $6.0 \mathrm{E}+06$ & $7.0 \mathrm{E}+06$ & $8.0 \mathrm{E}+06$ & $9.0 \mathrm{E}+06$ & $1.0 \mathrm{E}+07$ \\
\hline$\hat{p}_{\mathrm{f}}$ & $3.3936 \mathrm{E}-04$ & $3.3983 \mathrm{E}-04$ & $3.3977 \mathrm{E}-04$ & $3.3784 \mathrm{E}-04$ & $3.3875 \mathrm{E}-04$ \\
\hline$\hat{p}_{\mathrm{f}}-2 \sigma_{\hat{p}_{\mathrm{f}}}$ & $3.2434 \mathrm{E}-04$ & $3.2592 \mathrm{E}-04$ & $3.2676 \mathrm{E}-04$ & $3.2557 \mathrm{E}-04$ & $3.2711 \mathrm{E}-04$ \\
\hline$\hat{p}_{\mathrm{f}}+2 \sigma_{\hat{p}_{\mathrm{f}}}$ & $3.5438 \mathrm{E}-04$ & $3.5374 \mathrm{E}-04$ & $3.5278 \mathrm{E}-04$ & $3.5011 \mathrm{E}-04$ & $3.5039 \mathrm{E}-04$ \\
\hline
\end{tabular}

Table 2. Probability of failure estimated by MCS with different sample size

\begin{tabular}{|c|c|c|c|c|c|}
\hline$N$ & $\mathbf{1 . 0 E}+\mathbf{0 6}$ & $\mathbf{2 . 0 E}+\mathbf{0 6}$ & $\mathbf{3 . 0 E}+\mathbf{0 6}$ & $\mathbf{4 . 0 E}+\mathbf{0 6}$ & $\mathbf{5 . 0 E}+\mathbf{0 6}$ \\
\hline$\hat{p}_{\mathrm{f}}$ (LHS) & $3.3034 \mathrm{E}-04$ & $3.3924 \mathrm{E}-04$ & $3.3738 \mathrm{E}-04$ & $3.3891 \mathrm{E}-04$ & $3.3944 \mathrm{E}-04$ \\
\hline$N$ & $6.0 \mathrm{E}+06$ & $7.0 \mathrm{E}+06$ & $8.0 \mathrm{E}+06$ & $9.0 \mathrm{E}+06$ & $1.0 \mathrm{E}+07$ \\
\hline$\hat{p}_{\mathrm{f}}$ (LHS) & $3.4013 \mathrm{E}-04$ & $3.3893 \mathrm{E}-04$ & $3.4024 \mathrm{E}-04$ & $3.3812 \mathrm{E}-04$ & $3.3871 \mathrm{E}-04$ \\
\hline
\end{tabular}

Table 3. Probability of failure estimated by LHS with different sample size

\begin{tabular}{|c|c|c|c|c|c|}
\hline$N$ & $\mathbf{1 . 0 E}+\mathbf{0 6}$ & $\mathbf{2 . 0 E}+\mathbf{0 6}$ & $\mathbf{3 . 0 E}+\mathbf{0 6}$ & $\mathbf{4 . 0 E}+\mathbf{0 6}$ & $\mathbf{5 . 0 E}+\mathbf{0 6}$ \\
\hline$\hat{p}_{\mathrm{f}}$ (IS) & $3.3880 \mathrm{E}-04$ & $3.3881 \mathrm{E}-04$ & $3.3871 \mathrm{E}-04$ & $3.3874 \mathrm{E}-04$ & $3.3885 \mathrm{E}-04$ \\
\hline$N$ & $6.0 \mathrm{E}+06$ & $7.0 \mathrm{E}+06$ & $8.0 \mathrm{E}+06$ & $9.0 \mathrm{E}+06$ & $1.0 \mathrm{E}+07$ \\
\hline$\hat{p}_{\mathrm{f}}$ (IS) & $3.3884 \mathrm{E}-04$ & $3.3880 \mathrm{E}-04$ & $3.3884 \mathrm{E}-04$ & $3.3882 \mathrm{E}-04$ & $3.3878 \mathrm{E}-04$ \\
\hline
\end{tabular}

Table 4. Probability of failure estimated by IS with different sample size 


\begin{tabular}{|c|c|c|c|c|c|}
\hline \multirow{2}{*}{$\begin{array}{c}\text { Reliability } \\
\text { Method }\end{array}$} & \multicolumn{2}{|c|}{ Local Reliability Methods } & \multicolumn{3}{c|}{ Sampling Methods } \\
\cline { 2 - 6 } & FORM & SORM & MCS & LHS & IS \\
\hline $\begin{array}{c}\text { Failure } \\
\text { Probability }\end{array}$ & $3.2113 \mathrm{E}-04$ & $3.3869 \mathrm{E}-04$ & $3.3875 \mathrm{E}-04$ & $3.3871 \mathrm{E}-04$ & $3.3878 \mathrm{E}-04$ \\
\hline
\end{tabular}

Table 5. Results from different reliability methods

\section{Summary}

Sampling methods do not rely on the convergence of design point search or approximations of the true limit state. They are generally more accurate than these local reliability methods and independent with results obtained by FORM or SORM reliability analysis. However, sampling methods typically require a large number of performance function evaluations, which makes them impractical if the performance function is expensive to evaluate. With the variance reduction techniques, such as importance sampling, directional simulation and subset simulation described in this section, the number of sampling could be cut down in some degree. Even with the help of variance reduction techniques, if the performance function evaluations are performed through complex calculations, such as finite element simulations, which are computationally cost, sampling methods would be infeasible for computation burden problems.

\section{References}

[1] Bastidas-Arteaga E., \& Soubra A.H., Reliability Analysis Methods. ALERT Doctoral School 2014-Stochastic Analysis and Inverse Modelling, pp. 53-77, 2014.

[2] Nikolaidis E., Ghiocel D.M., \& Singhal S. (Eds.). Engineering design reliability applications: for the aerospace, automotive and ship industries. CRC Press, 2007.

[3] Mahadevan S., \& Haldar A., Probability, reliability and statistical method in engineering design. John Wiley \& Sons, 2000.

[4] Liu J.S., Monte Carlo strategies in scientific computing. Springer Science \& Business Media, 2008.

[5] Tokdar S.T., \& Kass R.E. Importance sampling: a review. Wiley Interdisciplinary Reviews: Computational Statistics, vol. 2, no. 1, pp. 54-60, 2010.

[6] Rubinstein R.Y., \& Kroese D.P., Simulation and the Monte Carlo method (Vol. 707). John Wiley \& Sons, 2011.

[7] Bucher C.G., Adaptive sampling — an iterative fast Monte Carlo procedure. Structural Safety, vol. 5, no. 2, 119-126, 1988.

[8] Lemaire M., Structural reliability. John Wiley \& Sons, 2013.

[9] Conover W.J., On a better method of selecting values of input variables for computer codes. Unpublished manuscript, 1975.

[10] McKay M. D., \& Beckman R.J., A Comparison of Three Methods for Selecting Values of Input Variables in the Analysis of Output from a Computer Code. TECHNOMETRICS, vol. 21, no. 2, 1979.

[11] Nie J., \& Ellingwood B.R., Directional methods for structural reliability analysis. Structural Safety, vol. 22, no. 3, pp. 233-249, 2000.

[12] Bjerager P., Methods for structural reliability computations. In Reliability problems: general principles and applications in mechanics of solids and structures (pp. 89-135). Springer Vienna, 1991.

[13] Melchers R.E., Structural system reliability assessment using directional simulation. Structural Safety, vol. 16, no. 1, pp. 23-37, 1994.

[14] Moarefzadeh M.R., \& Melchers R.E., Directional importance sampling for ill-proportioned spaces. Structural Safety, vol. 21, no. 1, pp. 1-22, 1999.

[15] Fajardo J.F., Perez A.A.J., Alsina S.S.M., \& Ramirez-Marquez J.E. (Eds.). Simulation methods for reliability and availability of complex systems. Springer Science \& Business Media, 2010.

[16] Au S.K., \& Beck J.L., Subset simulation and its application to seismic risk based on dynamic analysis. Journal of Engineering Mechanics, vol. 129, no. 8, pp. 901-917, 2003.

[17] Au S.K., \& Beck J.L., Estimation of small failure probabilities in high dimensions by subset simulation. Probabilistic Engineering Mechanics, vol. 16, no. 4, pp. 263-277, 2001. 